\title{
Atorvastatin Decreased Circulating RANTES Levels in Impaired Glucose Tolerance Patients with Hypercholesterolemia: An Interventional Study
}

\author{
Xiaomeng Feng · Xia Gao · Yumei Jia · Heng Zhang • \\ Yuan Xu
}

Received: November 19, 2016 / Published online: January 24, 2017

(c) The Author(s) 2017. This article is published with open access at Springerlink.com

\section{ABSTRACT}

Introduction: Impaired glucose tolerance (IGT) is the major cause of the development of both type 2 diabetes and atherosclerosis. Regulated upon activation, normal $\mathrm{T}$ cells expressed and secreted (RANTES), a proinflammatory chemokine, is associated with atherosclerosis. We investigated the effect of atorvastatin on circulating RANTES in IGT patients with hypercholesterolemia.

Methods: This study evaluated cross-sectional and interventional studies of 32 IGT patients with hypercholesterolemia (group A) and 32 controls (group B). Group A was treated with atorvastatin (20 mg/day) for 8 weeks. Platelet-free plasma (PFP) RANTES and clinical characteristics were examined.

Results: PFP RANTES was significantly higher in group A compared with group B $(9.76 \pm 3.10$ vs $6.43 \pm 2.16 \mathrm{ng} / \mathrm{ml}, P<0.001)$. PFP RANTES was positively correlated with total cholesterol (TC) $\quad(r=0.589, \quad P<0.001), \quad$ low-density lipoprotein cholesterol (LDL-C) $\quad(r=0.583$,

Enhanced content To view enhanced content for this article go to http://www.medengine.com/Redeem/ 2387F0601C68BFFA.

X. Feng · X. Gao · Y. Jia · H. Zhang · Y. Xu ( () Department of Endocrinology, Beijing Chao-Yang Hospital, Capital Medical University,

Beijing 100020, China

e-mail: xuyuan3200@sina.com
$P<0.001), \quad$ triglycerides $\quad(\mathrm{TG}) \quad(r=0.450$, $P<0.001)$, fasting blood glucose (FBG) $(r=0.469, \quad P<0.001), \quad 2$-hour postchallenge glucose $\quad(2 \mathrm{hPG}) \quad(r=0.397, \quad P=0.001)$, glycosylated hemoglobin (HbA1c) $(r=0.353$, $P=0.004)$, and high sensitivity C-reactive protein (hsCRP) $(r=0.616, \quad P<0.001)$, and negatively related to high-density lipoprotein cholesterol (HDL-C) $(r=-0.272, P=0.029)$. After controlling for confounders, LDL-C $(\beta=2.109, \quad P<0.001)$ and hsCRP $(\beta=0.272$, $P=0.029)$ were independently related to RANTES. After atorvastatin treatment, PFP RANTES significantly decreased in group A compared with baseline (from $9.76 \pm 3.10$ to $7.48 \pm 2.78 \mathrm{ng} / \mathrm{ml}, P<0.001)$.

Conclusions: Atorvastatin decreased circulating RANTES in IGT patients with hypercholesterolemia, indicating that statins may play an important role in inhibiting inflammatory responses in patients with IGT.

Keywords: Atorvastatin;

Hypercholesterolemia; Impaired glucose tolerance; Regulated upon activation, normal $\mathrm{T}$ cells expressed and secreted

\section{INTRODUCTION}

Diabetes is a cardiovascular risk equivalent, and atherosclerotic cardiovascular disease (ASCVD) is the major risk of death in patients with type 2 
diabetes. Recent studies have demonstrated that the risk of ASCVD begins to increase considerably before the onset of diabetes. Impaired glucose tolerance (IGT), characterized by high postprandial blood glucose levels, is the major cause of the development of both type 2 diabetes [1] and atherosclerosis [2]. The postprandial blood glucose levels are associated with the highest diurnal levels of glycemia and the greatest fluctuations in blood glucose levels that may have more damaging effects on the vasculature [3, 4], including activation of inflammatory pathways, elevated insulin resistance, increased oxidative stress, extensive procoagulant state, and abnormal vasomotion.

Atherosclerosis, a major risk factor for diabetic cardiovascular complications, is considered as a chronic disease characterized by inflammation. Chemokines are proinflammatory cytokines, which may lead to atherosclerosis and plaque destabilization not only by recruiting activated leukocytes into the lesion but also by directly contributing to plaque rupture and thrombus formation. In addition, inflammation might be crucial for insulin resistance and hyperglycemia [5]. Chemokines are also involved in the pathogenesis of insulin resistance which may cause metabolic syndrome and disorders of glycometabolism ultimately. Recent in vitro evidence has suggested that monocyte chemotactic protein 1 (MCP-1) induces insulin resistance in both adipocytes and skeletal muscle cells [6]. Regulated upon activation, normal $\mathrm{T}$ cells expressed and secreted (RANTES), also known as $\mathrm{C}-\mathrm{C}$ chemokine ligand 5 (CCL5), belongs to the $\mathrm{C}-\mathrm{C}$ chemokine family, which is secreted by many cell types such as endothelial cells, smooth muscle cells, macrophages, platelets, and activated T cells. RANTES plays a critical role in the chronic inflammatory processes and the progression of atherosclerosis [7]. Moreover, RANTES has been shown to affect glycometabolism and insulin resistance. Based on our previous studies, high RANTES levels were reported in type 2 diabetes patients with hypertriglyceridemia compared with controls [8]. Therefore, RANTES administration has been proposed as a potential therapeutic strategy to protect the cardiovascular system [9-11]; thus, it may have implications for decreasing cardiovascular risk of diabetes and prediabetes.

Statins, the 3-hydroxy-3-methylglutaryl coenzyme A (HMG-CoA) reductase inhibitors, are widely used in clinical practice as low-density lipoprotein cholesterol (LDL-C)-lowering agents, which have been conclusively demonstrated to have benefits in primary $[12,13]$ and secondary $[14,15]$ prevention of cardiovascular diseases. In addition to lipid-lowering effects, statins have been documented to induce favorable effects on regulating vascular redox state, improving endothelial function, suppressing platelet activation, promoting plaque stabilization, and inhibiting $\mathrm{T}$ cell activation, macrophage infiltration, and vascular wall inflammation [16]. Therefore, the anti-inflammatory effect of statins has been considered responsible for their protective effects by some clinical researchers [12, 14, 17-19].

Although statins were proven to reduce cardiovascular complications in patients with diabetes [20], only few reports about the regulation of the inflammatory processes by statins in patients with prediabetes have been published. To the best of our knowledge, the effects of statins on circulating RANTES levels in patients with impaired glucose tolerance have not been well characterized. In this study, we aimed to examine whether atorvastatin modulated platelet-free plasma (PFP) RANTES levels in the impaired glucose tolerance patients with hypercholesterolemia.

\section{METHODS}

\section{Subjects}

All participants (both genders) ranging in age from 30 to 70 years were recruited from December 2014 to March 2015, and $75 \mathrm{~g}$ oral glucose tolerance tests (OGTT) were performed at screening.

Thirty-two impaired glucose tolerance patients with hypercholesterolemia (group A) were recruited for this study from a group of 
outpatients at the Department of Endocrinology, Beijing Chao-Yang Hospital, Capital Medical University, Beijing, China. Patients diagnosed with IGT, as defined by the American Diabetes Association criteria, were eligible for the study. Additionally, the LDL-C levels of participants were greater than $2.6 \mathrm{mmol} / \mathrm{l}$. The following exclusion criteria for group A were applied: normal glucose tolerance, impaired fasting glucose, diabetes, and contraindicating treatment with atorvastatin.

Thirty-two healthy people (group B) were recruited as the control group from the community or from the group of people undergoing routine medical checkups. None of them had a history of prediabetes (including impaired glucose tolerance and impaired fasting glucose), diabetes, or hyperlipidemia.

Moreover, people with hypertension, coronary artery disease, endocrine disease, systemic inflammatory disease, infectious disease, cancer, chronic kidney disease [i.e., creatinine (CR) greater than $120 \mu \mathrm{mol} / \mathrm{l}$ ], hepatic enzymes [i.e., aspartate aminotransferase (AST) and alanine aminotransferase (ALT)] greater than 1.5 times the upper normal limits, creatine kinase (CK) greater than 1.5 times the upper normal limit, a history of alcohol abuse, using heparin within the last 3 months, pregnancy and lactation were excluded from both groups. People taking agents known to influence glucose or insulin metabolism, and/or people being treated with lipid-lowering drugs were also excluded from both groups.

\section{Study Design}

Participants in group A were required to attend three study visits: the screening visit, visit 1 , and visit 2 (spaced 8 weeks apart), while participants in group B attended the screening visit. Starting at visit 1, the group A participants who fulfilled the inclusion criteria (without any exclusion criterion) were administered atorvastatin $20 \mathrm{mg} /$ day for 8 weeks. The tablets were counted at visit 2 , and compliance was considered to be satisfactory if more than $90 \%$ of tablets were taken.

Blood samples and the data on the medical history, height, weight, and blood pressure were collected at the screening visit (groups A and B) and at visit 2 (group A) (under fasting conditions, as described below). At visit 1, each participant in group A received instructions to maintain his/her usual nutritional and exercise habits. Participants in group A were asked to immediately report the development of unusual muscle soreness or pain throughout the study. In addition, any adverse event in each group A participant was recorded at visit 2 .

\section{Compliance with Ethics Guidelines}

The study was approved by the Medicine and Pharmacy Ethics Committee of Beijing Chao-Yang Hospital, Capital Medical University, Beijing, China. All procedures followed were in accordance with the ethical standards of the responsible committee on human experimentation (institutional and national) and with the 1964 Declaration of Helsinki, as revised in 2013. Informed consent was obtained from all participants for being included in the study.

\section{Data Collection and Laboratory Tests}

A complete medical history, including duration and treatment of any disease, was obtained from each participant; height and weight were determined using a standardized protocol. Body mass index (BMI) was calculated as weight $(\mathrm{kg}) /$ [height $(\mathrm{m})]^{2}$. Blood pressure was measured using a calibrated standard mercury sphygmomanometer. All readings were measured after a 5-min rest, with the patients in the sitting position.

Fasting blood samples were collected in the morning after an 8-h overnight fast. Total cholesterol (TC), high-density lipoprotein cholesterol (HDL-C), LDL-C, triglycerides (TG), fasting blood glucose (FBG), 2-hour postchallenge glucose (2hPG), high sensitivity C-reactive protein (hsCRP), AST, ALT, CR, CK, and glycosylated hemoglobin (HbA1c) were measured in the central laboratory of Beijing Chao-Yang Hospital, Capital Medical University. 
Blood samples for preparation of platelet-free plasma from all participants were collected through standard phlebotomy into citrate tubes. They were promptly placed on ice after blood collection and were centrifuged within $30 \mathrm{~min}$ at $4{ }^{\circ} \mathrm{C}$ for $10 \mathrm{~min}$ at the relative centrifugal force (RCF) of $11,000 \mathrm{~g}$. Each supernatant was isolated using a plastic transfer pipet, taking care not to disturb the plasma in the bottom of the tube, and was placed in a plastic centrifuge tube with a cap. The platelet-free plasma samples were stored at $-80{ }^{\circ} \mathrm{C}$ within $2 \mathrm{~h}$ after blood collection; they should not be thawed until analyses. RANTES concentrations were measured in duplicate at the same time using enzyme-linked immunosorbent assay (ELISA) kits (R\&D Systems, Minneapolis, MN, USA) with CV $3.5 \%$ for intraobserver and $8.4 \%$ for interobserver for quantitative detection with an automated ELISA reader (VARIOSKAN FLASH-5250040, Thermo Scientific, USA).

Adverse events were recorded throughout the study. The safety parameters included AST, ALT, CR, and CK.

\section{Statistical Analysis}

All analyses were performed with Statistical Package for Social Sciences version 19.0 (SPSS, Inc, Chicago, IL, USA). Normally distributed data were expressed as the mean \pm standard deviation (SD). Non-normally distributed data were given as medians (25th and 75th percentiles). Comparisons of the baseline clinical and biochemical markers, as well as the RANTES levels, between groups A and B were performed using independent sample $t$ tests and Mann-Whitney $U$ Tests. Comparisons of the pretreatment and posttreatment (with atorvastatin) clinical and biochemical markers, as well as the RANTES levels, in group A were performed with paired $t$ tests and Wilcoxon tests. Proportions were analyzed using the Chi-squared test. The association between the baseline values of RANTES and the other baseline parameters was examined using Pearson's and Spearman's correlation coefficient analyses. Variables with a $P$ value less than 0.05 in Pearson's and Spearman's correlation coefficient analyses were retained for the multiple stepwise regression analysis. In all statistical tests, $P$ values less than 0.05 were considered to be significant, and all tests were two-sided.

\section{RESULTS}

\section{Baseline Clinical Characteristics of the Study Participants}

The baseline clinical characteristics of the study participants are listed in Table 1. The participants in the two groups were similar in sex, age, BMI, systolic blood pressure (SBP), and diastolic blood pressure (DBP) $(P>0.05$ for all). The levels of TC, LDL-C, TG, FBG, 2hPG, HbA1c, and hsCRP were higher and the levels of HDL-C were lower in group A compared with group B $(P<0.01$ for all).

\section{Baseline RANTES Levels of the Study Participants}

The fasting PFP RANTES levels were significantly higher in group A than in group B $(9.76 \pm 3.10$ vs $6.43 \pm 2.16 \mathrm{ng} / \mathrm{ml}, P<0.001)$ (Fig. 1).

\section{Correlation Between RANTES Levels and the Baseline Parameters}

The following parameters were found to be positively correlated with the PFP RANTES levels: TC, LDL-C, TG, FBG, 2hPG, HbA1c, and hsCRP $(P<0.01$ for all). In addition, HDL-C was negatively related to PFP RANTES levels $(P<0.05)$ (Table 2). After adjusting for the confounders, the multiple stepwise regression analysis showed that only increased LDL-C $(P<0.001)$ and hsCRP $(P=0.029)$ levels were independently related to high PFP RANTES 
Table 1 Baseline clinical characteristics of the study participants

\begin{tabular}{lllr}
\hline Parameters & Group A $(\boldsymbol{n}=\mathbf{3 2})$ & Group B $(\boldsymbol{n}=32)$ & P value \\
\hline Sex $(\mathrm{M} / \mathrm{F})$ & $19 / 13$ & $15 / 17$ & 0.316 \\
Age $($ years $)$ & $56.97 \pm 8.89$ & $55.13 \pm 7.02$ & 0.361 \\
BMI $\left(\mathrm{kg} / \mathrm{m}^{2}\right)$ & $26.11 \pm 4.57$ & $24.84 \pm 3.60$ & 0.222 \\
SBP $(\mathrm{mmHg})$ & $126.50 \pm 5.17$ & $124.60 \pm 8.97$ & 0.303 \\
DBP $(\mathrm{mmHg})$ & $75.56 \pm 8.70$ & $72.72 \pm 7.38$ & 0.164 \\
TC $(\mathrm{mmol} / \mathrm{l})$ & $5.57 \pm 0.88$ & $4.28 \pm 0.52$ & $<0.001$ \\
HDL-C $(\mathrm{mmol} / \mathrm{l})$ & $1.34 \pm 0.24$ & $1.63 \pm 0.36$ & $<0.001$ \\
LDL-C $(\mathrm{mmol} / \mathrm{l})$ & $3.40 \pm 0.64$ & $2.20 \pm 0.30$ & $<0.001$ \\
TG $(\mathrm{mmol} / \mathrm{l})$ & $1.15(0.98,2.04)$ & $0.69(0.50,0.88)$ & $<0.001$ \\
FBG $(\mathrm{mmol} / \mathrm{l})$ & $6.44 \pm 0.29$ & $4.99 \pm 0.35$ & $<0.001$ \\
2hPG $(\mathrm{mmol} / \mathrm{l})$ & $9.31 \pm 0.82$ & $6.43 \pm 0.74$ & $<0.001$ \\
HbAlc $(\%)$ & $6.20 \pm 0.31$ & $5.46 \pm 0.33$ & $<0.001$ \\
hsCRP $(\mathrm{mg} / \mathrm{l})$ & $1.84(1.02,3.26)$ & $1.15(0.78,1.63)$ & 0.009 \\
\hline
\end{tabular}

Group $A$ impaired glucose tolerance patients with hypercholesterolemia, Group $B$ control subjects, BMI body mass index, $S B P$ systolic blood pressure, $D B P$ diastolic blood pressure, $T C$ total cholesterol, $H D L$ - $C$ high-density lipoprotein cholesterol, $L D L-C$ low-density lipoprotein cholesterol, $T G$ triglycerides, $F B G$ fasting blood glucose, $2 h P G$ 2-hour postchallenge glucose, $H b A 1 c$ glycosylated hemoglobin, $b S C R P$ high sensitivity $\mathrm{C}$-reactive protein

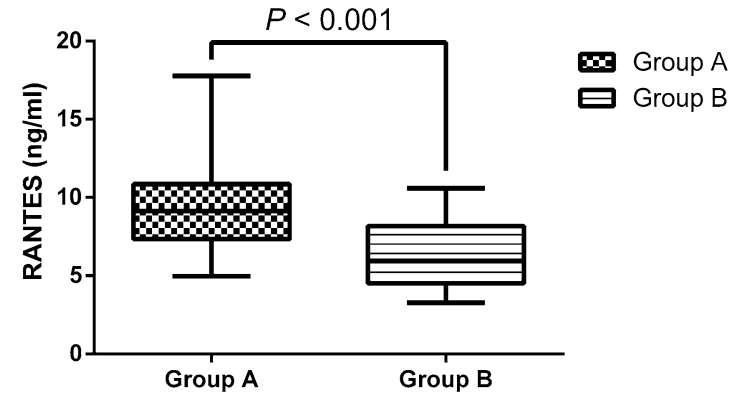

Fig. 1 Baseline platelet-free plasma regulated upon activation, normal $\mathrm{T}$ cells expressed and secreted (RANTES) levels in the study participants. The values are expressed as medians (25th and 75th percentiles). Group A: impaired glucose tolerance patients with hypercholesterolemia $(n=32)$; Group B: control subjects $(n=32)$

levels (Table 3). The multiple regression equation was $Y_{\text {RANTES }}=1.601+2.109 X_{\text {LDL-C }}$ $+0.272 X_{\text {hsCRP. }}$ The model had an adjusted $R$ squared of $0.370, F=19.500$, and $P<0.001$.

\section{Effects of Atorvastatin on the Clinical Characteristics in Group A}

The pretreatment and posttreatment (with atorvastatin) clinical parameters in group A are summarized in Table 4. Compared with baseline, at visit 2 the patients in group A presented significantly lower levels of TC, LDL-C, and hsCRP $(P<0.01$ for all $)$. In addition, no statistically significant changes were observed in BMI, SBP, DBP, HDL-C, TG, FBG, AST, ALT, CR, and $\mathrm{CK}$ after 8 weeks of atorvastatin treatment compared with baseline ( $P>0.05$ for all).

\section{Effect of Atorvastatin on the Levels of RANTES in Group A}

After 8 weeks of atorvastatin treatment, the PFP RANTES levels in group A were significantly decreased compared with the baseline levels (from $9.76 \pm 3.10 \mathrm{ng} / \mathrm{ml}$ at pretreatment to $7.48 \pm$ $2.78 \mathrm{ng} / \mathrm{ml}$ at posttreatment, $P<0.001$ ) (Fig. 2). 


\section{Safety Parameters}

All participants completed the study, and no serious adverse effects were observed throughout the study.

Table 2 Correlation analyses of the baseline parameters associated with RANTES

\begin{tabular}{lrr}
\hline Parameters & \multicolumn{1}{l}{$r$} & $\boldsymbol{P}$ value \\
\hline Age $($ years $)$ & 0.014 & 0.914 \\
BMI $\left(\mathrm{kg} / \mathrm{m}^{2}\right)$ & 0.212 & 0.092 \\
SBP $(\mathrm{mmHg})$ & 0.087 & 0.493 \\
DBP $(\mathrm{mmHg})$ & 0.132 & 0.299 \\
TC $(\mathrm{mmol} / \mathrm{l})$ & 0.589 & $<0.001$ \\
HDL-C $(\mathrm{mmol} / \mathrm{l})$ & -0.272 & 0.029 \\
LDL-C (mmol/l) & 0.583 & $<0.001$ \\
TG $(\mathrm{mmol} / \mathrm{l})$ & 0.450 & $<0.001$ \\
FBG $(\mathrm{mmol} / \mathrm{l})$ & 0.469 & $<0.001$ \\
2hPG $(\mathrm{mmol} / \mathrm{l})$ & 0.397 & 0.001 \\
HbAlc $(\%)$ & 0.353 & 0.004 \\
hsCRP $(\mathrm{mg} / \mathrm{l})$ & 0.616 & $<0.001$ \\
\hline
\end{tabular}

RANTES regulated upon activation, normal $\mathrm{T}$ cells expressed and secreted, $B M I$ body mass index, $S B P$ systolic blood pressure, $D B P$ diastolic blood pressure, $T C$ total cholesterol, $H D L-C$ high-density lipoprotein cholesterol, $L D L-C$ low-density lipoprotein cholesterol, $T G$ triglycerides, $F B G$ fasting blood glucose, $2 h P G$ 2-hour postchallenge glucose, HbAlc glycosylated hemoglobin, bs CRP high sensitivity $\mathrm{C}$-reactive protein

\section{DISCUSSION}

In this study, we demonstrated that PFP RANTES levels were significantly higher in the impaired glucose tolerance patients with hypercholesterolemia compared with the controls. This finding is similar to our previous results that RANTES levels were significantly higher in type 2 diabetes patients with hypertriglyceridemia compared with controls [8], and aligns with some other studies indicating that circulating RANTES levels were significantly higher in patients with dyslipidemia [21], hyperglycemia [22, 23], or metabolic syndrome [24] compared with controls. Our present study also documented that PFP RANTES levels were positively correlated with the levels of TC, TG, FBG, $2 \mathrm{hPG}$, and $\mathrm{HbA} 1 \mathrm{c}$, but negatively related to the levels of HDL-C, which supported that RANTES might be associated with disorders of metabolism. In particular, our finding that increased LDL-C and hsCRP, major predictors for cardiovascular events, were independently related to high PFP RANTES levels after controlling for confounders suggested that RANTES might play an important role in inflammatory processes and cardiovascular events.

Importantly, we report for the first time here that atorvastatin treatment administered to the impaired glucose tolerance patients with hypercholesterolemia for 8 weeks resulted in a significant decrease in PFP RANTES levels, suggesting that statins might cause the inhibition of cardiovascular diseases,

Table 3 Multiple regression analysis of the baseline parameters associated with RANTES

\begin{tabular}{lllllr}
\hline Parameters & $\boldsymbol{\beta}$ & SE & Standardized $\boldsymbol{\beta}$ & 95\% CI & $\boldsymbol{P}$ value \\
\hline Constant & 1.601 & 1.172 & & -0.743 to 3.945 & 0.177 \\
LDL-C (mmol/l) & 2.109 & 0.417 & 0.523 & $1.275-2.942$ & $<0.001$ \\
hsCRP $(\mathrm{mmol} / \mathrm{l})$ & 0.272 & 0.122 & 0.232 & $0.029-0.515$ & 0.029 \\
\hline
\end{tabular}

Adjustment for the variables with $P<0.05$ in Pearson's and Spearman's correlation coefficient analyses: total cholesterol (TC), high-density lipoprotein cholesterol (HDL-C), LDL-C, triglycerides (TG), fasting blood glucose (FBG), 2-hour postchallenge glucose (2hPG), glycosylated hemoglobin (HbAlc), and hsCRP

RANTES regulated upon activation, normal T cells expressed and secreted, $S E$ standard error, $C I$ confidence interval, $L D L-C$ low-density lipoprotein cholesterol, hsCRP high sensitivity C-reactive protein 
Table 4 Pretreatment and posttreatment clinical characteristics of the impaired glucose tolerance patients with hypercholesterolemia treated with atorvastatin

\begin{tabular}{lllr}
\hline Parameters & Pretreatment $(\boldsymbol{n}=\mathbf{3 2})$ & Posttreatment $(\boldsymbol{n}=32)$ & $\boldsymbol{P}$ value \\
\hline BMI $\left(\mathrm{kg} / \mathrm{m}^{2}\right)$ & $26.11 \pm 4.57$ & $26.03 \pm 4.57$ & 0.244 \\
SBP $(\mathrm{mmHg})$ & $126.50 \pm 5.17$ & $125.63 \pm 5.46$ & 0.255 \\
DBP $(\mathrm{mmHg})$ & $75.56 \pm 8.07$ & $73.84 \pm 8.06$ & 0.151 \\
TC $(\mathrm{mmol} / \mathrm{l})$ & $5.57 \pm 0.88$ & $4.27 \pm 1.25$ & $<0.001$ \\
HDL-C $(\mathrm{mmol} / \mathrm{l})$ & $1.34 \pm 0.24$ & $1.58 \pm 0.78$ & 0.091 \\
LDL-C $(\mathrm{mmol} / \mathrm{l})$ & $3.40 \pm 0.64$ & $2.37 \pm 0.93$ & $<0.001$ \\
TG $(\mathrm{mmol} / \mathrm{l})$ & $1.51(0.98,2.04)$ & $1.18(0.81,1.64)$ & 0.060 \\
FBG $(\mathrm{mmol} / \mathrm{l})$ & $6.44 \pm 0.29$ & $6.23 \pm 0.54$ & 0.078 \\
hsCRP $(\mathrm{mg} / \mathrm{l})$ & $1.84(1.02,3.26)$ & $1.36(0.38,2.16)$ & 0.002 \\
AST $(\mathrm{U} / \mathrm{l})$ & $20.34 \pm 5.68$ & $20.67 \pm 8.76$ & 0.836 \\
ALT $(\mathrm{U} / \mathrm{l})$ & $23.53 \pm 9.92$ & $22.41 \pm 10.67$ & 0.638 \\
CR $(\mu \mathrm{mol} / \mathrm{l})$ & $63.68 \pm 14.84$ & $66.04 \pm 16.05$ & 0.515 \\
CK $(\mathrm{U} / \mathrm{l})$ & $83.22 \pm 27.81$ & $86.81 \pm 36.39$ & 0.487 \\
\hline
\end{tabular}

$B M I$ body mass index, $S B P$ systolic blood pressure, $D B P$ diastolic blood pressure, $T C$ total cholesterol, $H D L$ - $C$ high-density lipoprotein cholesterol, $L D L-C$ low-density lipoprotein cholesterol, $T G$ triglycerides, $F B G$ fasting blood glucose, $h s C R P$ high sensitivity C-reactive protein, $A S T$ aspartate aminotransferase, $A L T$ alanine aminotransferase, $C R$ creatinine, $C K$ creatine kinase

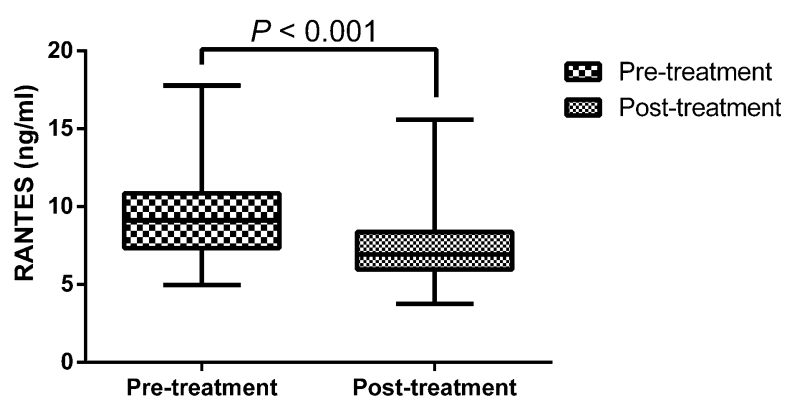

Fig. 2 Platelet-free plasma regulated upon activation, normal $\mathrm{T}$ cells expressed and secreted (RANTES) levels in the impaired glucose tolerance patients with hypercholesterolemia after 8 weeks of atorvastatin treatment compared with the baseline levels. The values are expressed as medians (25th and 75th percentiles) $(n=32)$

independent of their effects on the reduction in LDL-C.

Statins have been reported to have beneficial anti-inflammatory effects apart from lowing LDL-C levels by some large-scale research studies. The pravastatin treatment significantly reduced C-reactive protein (CRP) levels in subjects with or without cardiovascular diseases, independent of any changes in LDL-C [17]. Later studies extended these findings and established that patients who achieved both reduced LDL-C levels and decreased inflammatory mediators benefited the best in the prevention of cardiovascular events through statin treatment [18]. Furthermore, statin treatment was shown to remain effective at alleviating risk in subjects with high CRP but low LDL-C [13, 19]. Other smaller clinical studies have also demonstrated anti-inflammatory properties of statins: they mitigated CRP and circulating proinflammatory cytokine levels in patients with hypercholesterolemia [25], diabetes mellitus [26], or metabolic syndrome [27].

Recent evidence has indicated that RANTES, a proinflammatory chemokine, is associated with cardiovascular events and hyperglycemia. 
However, the data have been inconsistent. Most studies have supported that the elevated RANTES levels are related to high cardiovascular risk. Increased levels of RANTES were reported in patients with acute coronary syndromes compared with controls [28] and were proven in patients with refractory ischemic symptoms compared with stabilized patients [29]. RANTES levels were associated with carotid wall thickness and lipid-core volume [30], suggesting that high RANTES levels might be related to extensive carotid atherosclerosis and plaque at high risk of rupturing. Therefore, the inhibition of RANTES is supposed to cause cardioprotective effects through its anti-inflammatory capacities. By its ability to bind to its chemokine receptors expressed on T cells or monocytes, RANTES might contribute to the adhesion and the transmigration of $T$ cells and monocytes through the endothelial wall [31]; thus, blocking these receptors by injecting RANTES receptor antagonists could alleviate atherosclerosis $[9,10]$ and might attenuate the infarct size [12]. Controversially, there have been some studies indicating that low RANTES levels might be correlated with atherosclerosis $[32,33]$. It might be hypothesized that the decreased circulating RANTES levels in patients with high risk of cardiovascular events could reflect increased deposition of RANTES on the vascular endothelium leading to more RANTES receptor stimulation. In addition, RANTES has been involved in developing abnormal glucose metabolism. Patients with IGT or with type 2 diabetes presented increased RANTES levels compared with controls [8, 22, 23]. Moreover, the high levels of RANTES were related to the development of type 2 diabetes independently of metabolic syndrome-related risk factors [22]. These studies might support the idea that diabetes and prediabetes are the major cause of ASCVD. PPAR- $\alpha$ agonists have been reported to decrease RANTES expression in vitro and animal studies [34-37]. Furthermore, our previous study demonstrated that RANTES levels reduced through fenofibrate treatment in type 2 diabetes mellitus patients with hypertriglyceridemia [8]. Although there was a study showing that simvastatin did not reduce RANTES levels in obesity without comorbidities [38], most studies have supported that statins might reduce RANTES levels. Statins have been found to alleviate inflammatory cell infiltration in the arterial wall by attenuating RANTES expression in animal studies apart from the beneficial effects on cholesterol metabolism $[39,40]$. In clinical studies, statins might also reduce circulating RANTES levels in patients with coronary artery disease [41]. It is possible that these conflicting data are caused by the poly-pharmacotherapy or other confounding variables of the study populations, such as age, sex, and species of the subjects. These discrepancies might also be due to differences in the assays used by the different studies. Hence, the administration of statins could exert beneficial effects on inhibiting inflammatory responses and protecting cardiovascular system through alleviating inflammatory mediators such as RANTES.

Therefore, the reduction of circulating RANTES levels in the impaired glucose tolerance patients with hypercholesterolemia through atorvastatin treatment in the present study may partially explain the beneficial effects of statin therapy in clinical trials in which the favorable effects only partly correlated with cholesterol changes, although further animal and clinical studies are still needed to investigate the mechanism by which statins protect against the cardiovascular complications of hyperglycemia.

Our study had some limitations. Firstly, our study population was limited to Chinese. Therefore, our findings may not be directly applicable to other populations. Secondly, our sample size was relatively small so that our findings might not be powerful enough to account for potentially confounding factors in our analysis, and our results could be improperly influenced by some outliers due to the sample size. However, we performed the post hoc sample size calculation (with $G^{*}$ Power 3.1.9.2) showing that the power to compare the baseline RANTES levels between the impaired 
glucose tolerance patients with hypercholesterolemia and the controls was 1.00 , and that the power to compare the pretreatment and posttreatment (with atorvastatin) RANTES levels in the impaired glucose tolerance patients with hypercholesterolemia was $1.00 \quad(\alpha=0.05$, two-sided test). Additionally, a small sample size may result in a type II error in the statistical analysis, but we have demonstrated that atorvastatin decreased RANTES levels in the impaired glucose tolerance patients with hypercholesterolemia in the present study. Therefore, the power of the analyses might be sufficient in our study. Thirdly, because this was not a crossover study, we could not determine a causal relationship; however, it certainly raises credible hypotheses to be confirmed and extended by future prospective cohort and mechanistic studies. Finally, one should acknowledge that long-term follow-up will be necessary to evaluate whether statin treatment delays the progression of cardiovascular disease in patients with impaired glucose tolerance eventually. Despite these limitations, our results still provide strong evidence for the effectiveness of atorvastatin in reducing circulating RANTES levels in the impaired glucose tolerance patients with hypercholesterolemia.

\section{CONCLUSION}

We found a significant increase in circulating RANTES, a proinflammatory chemokine, in the impaired glucose tolerance patients with hypercholesterolemia. More importantly, we presented novel data that atorvastatin treatment significantly attenuated circulating RANTES levels in the impaired glucose tolerance patients with hypercholesterolemia. These results indicate that statin therapy plays a key role in preventing inflammatory responses in patients with impaired glucose tolerance. The physiologic and pathologic significance of our findings remain to be further elucidated.

\section{ACKNOWLEDGEMENTS}

This study was supported by grants from the Principal Research Project of Capital Medical University (No. 2016JYY130), the Undergraduate Scientific Researching Innovation Project of Capital Medical University (No. XSKY2016143), and the Research Project of Beijing Chao-Yang Hospital for Youth to Xiaomeng Feng. No funding or sponsorship was received for the article processing charges.

All named authors meet the International Committee of Medical Journal Editors (ICMJE) criteria for authorship for this manuscript, take responsibility for the integrity of the work as a whole, and have given final approval to the version to be published.

Disclosures. Xiaomeng Feng, Xia Gao, Yumei Jia, Heng Zhang, and Yuan $\mathrm{Xu}$ have nothing to disclose.

Compliance with Ethics Guidelines. The study was approved by the Medicine and Pharmacy Ethics Committee of Beijing Chao-Yang Hospital, Capital Medical University, Beijing, China. All procedures followed were in accordance with the ethical standards of the responsible committee on human experimentation (institutional and national) and with the 1964 Declaration of Helsinki, as revised in 2013. Informed consent was obtained from all participants for being included in the study.

Data Availability. The datasets during and/ or analyzed during the current study are available from the corresponding author on reasonable request.

Open Access. This article is distributed under the terms of the Creative Commons Attribution-NonCommercial 4.0 International License (http://creativecommons.org/licenses/ by-nc/4.0/), which permits any noncommercial use, distribution, and reproduction in any medium, provided you give appropriate 
credit to the original author(s) and the source, provide a link to the Creative Commons license, and indicate if changes were made.

\section{REFERENCES}

1. Gerstein HC, Santaguida P, Raina P, et al. Annual incidence and relative risk of diabetes in people with various categories of dysglycemia: a systematic overview and meta-analysis of prospective studies. Diabetes Res Clin Pract. 2007;78:305-12.

2. Tominaga M, Eguchi H, Manaka H, Igarashi K, Kato $\mathrm{T}$, Sekikawa A. Impaired glucose tolerance is a risk factor for cardiovascular disease, but not impaired fasting glucose: the Funagata Diabetes Study. Diabetes Care. 1999;22:920-4.

3. Ceriello A. Impaired glucose tolerance and cardiovascular disease: the possible role of post-prandial hyperglycemia. Am Heart J. 2004;147:803-7.

4. Cavalot F, Petrelli A, Traversa M, et al. Postprandial blood glucose is a stronger predictor of cardiovascular events than fasting blood glucose in type 2 diabetes mellitus, particularly in women: lessons from the San Luigi Gonzaga Diabetes Study. J Clin Endocrinol Metab. 2006;91:813-9.

5. Pickup JC. Inflammation and activated innate immunity in the pathogenesis of type 2 diabetes. Diabetes Care. 2004;27:813-23.

6. Sell H, Eckel J. Monocyte chemotactic protein-1 and its role in insulin resistance. Curr Opin Lipidol. 2007;18:258-62.

7. Charo IF, Ransohoff RM. The many roles of chemokines and chemokine receptors in inflammation. N Engl J Med. 2006;354:610-21.

8. Feng X, Gao X, Jia Y, Zhang H, Yu Y, Wang G. PPAR- $\alpha$ agonist fenofibrate decreased serum RANTES levels in type 2 diabetes patients with hypertriglyceridemia. Med Sci Monit. 2016;5:743-51.

9. Veillard NR, Kwak B, Pelli G, et al. Antagonism of RANTES receptors reduces atherosclerotic plaque formation in mice. Circ Res. 2004;94:253-61.

10. Cipriani S, Francisci D, Mencarelli A, et al. Efficacy of the CCR5 antagonist maraviroc in reducing early, ritonavir-induced atherogenesis and advanced plaque progression in mice. Circulation. 2013;127:2114-24.
11. Braunersreuther V, Pellieux C, Pelli G, et al. Chemokine CCL5/RANTES inhibition reduces myocardial reperfusion injury in atherosclerotic mice. J Mol Cell Cardiol. 2010;48:789-98.

12. Ridker PM, Rifai N, Clearfield M, et al. Measurement of C-reactive protein for the targeting of statin therapy in the primary prevention of acute coronary events. N Engl J Med. 2001;344:1959-65.

13. Shepherd J, Cobbe SM, Ford I, et al. Prevention of coronary heart disease with pravastatin in men with hypercholesterolemia. West of Scotland Coronary Prevention Study Group. N Engl J Med. 1995;333:1301-7.

14. Ridker PM, Rifai N, Pfeffer MA, Sacks F, Braunwald E. Long-term effects of pravastatin on plasma concentration of C-reactive protein. The Cholesterol and Recurrent Events (CARE) Investigators. Circulation. 1999;100:230-5.

15. The Long-Term Intervention with Pravastatin in Ischaemic Disease (LIPID) Study Group. Prevention of cardiovascular events and death with pravastatin in patients with coronary heart disease and a broad range of initial cholesterol levels. $\mathrm{N}$ Engl J Med. 1998;339:1349-57.

16. Antonopoulos AS, Margaritis M, Lee R, Channon K, Antoniades C. Statins as anti-inflammatory agents in atherogenesis: molecular mechanisms and lessons from the recent clinical trials. Curr Pharm Des. 2012;18:1519-30.

17. Albert MA, Danielson E, Rifai N, Ridker SM. Effect of statin therapy on C-reactive protein levels: the pravastatin inflammation/CRP evaluation (PRINCE): a randomized trial and cohort study. JAMA. 2001;286:64-70.

18. Ridker PM, Cannon CP, Morrow D, et al. C-reactive protein levels and outcomes after statin therapy. N Engl J Med. 2005;352:20-8.

19. Ridker PM, Danielson E, Fonseca FA, et al. Rosuvastatin to prevent vascular events in men and women with elevated C-reactive protein. N Engl J Med. 2008;359:2195-207.

20. Mortensen MB, Kulenovic I, Falk E. Statin use and cardiovascular risk factors in diabetic patients developing a first myocardial infarction. Cardiovasc Diabetol. 2016;15:81.

21. Wooten JS, Nambi P, Gillard BK, et al. Intensive lifestyle modification reduces Lp-PLA2 in dyslipidemic HIV/HAART patients. Med Sci Sports Exerc. 2013;45:1043-50.

22. Herder C, Haastert B, Müller-Scholze S, et al. Association of systemic chemokine concentrations 
with impaired glucose tolerance and type 2 diabetes: results from the Cooperative Health Research in the Region of Augsburg Survey S4 (KORA S4). Diabetes. 2005;2:S11-7.

23. Dworacka M, Krzyżagórska E, Iskakova S, Bekmukhambetov Y, Urazayev O, Dworacki G. Increased circulating RANTES in type 2 diabetes. Eur Cytokine Netw. 2014;25:46-51.

24. Ueba $\mathrm{T}$, Nomura $\mathrm{S}$, Inami $\mathrm{N}$, Yokoi $\mathrm{T}$, Inoue $\mathrm{T}$. Elevated RANTES level is associated with metabolic syndrome and correlated with activated platelets associated markers in healthy younger men. Clin Appl Thromb Hemost. 2014;20:813-8.

25. Ascer E, Bertolami MC, Venturinelli ML, et al. Atorvastatin reduces proinflammatory markers in hypercholesterolemic patients. Atherosclerosis. 2004;177:161-6.

26. van de Ree MA, Huisman MV, Princen HM, Meinders AE, Kluft C. Strong decrease of high sensitivity C-reactive protein with high-dose atorvastatin in patients with type 2 diabetes mellitus. Atherosclerosis. 2003;166:129-35.

27. Bulcao C, Ribeiro-Filho FF, Sanudo A, Roberta Ferreira SG. Effects of simvastatin and metformin on inflammation and insulin resistance in individuals with mild metabolic syndrome. Am J Cardiovasc Drugs. 2007;7:219-24.

28. Nomura S, Uehata S, Saito S, Osumi K, Ozeki Y, Kimura Y. Enzyme immunoassay detection of platelet-derived microparticles and RANTES in acute coronary syndrome. Thromb Haemost. 2003;89:506-12.

29. Kraaijeveld AO, de Jager SC, de Jager WJ, et al. CC chemokine ligand-5 (CCL5/RANTES) and CC chemokine ligand-18 (CCL18/PARC) are specific markers of refractory unstable angina pectoris and are transiently raised during severe ischemic symptoms. Circulation. 2007;116:1931-41.

30. Virani SS, Nambi V, Hoogeveen $R$, et al. Relationship between circulating levels of RANTES (regulated on activation, normal T-cell expressed, and secreted) and carotid plaque characteristics: the Atherosclerosis Risk in Communities (ARIC) Carotid MRI Study. Eur Heart J. 2011;32:459-68.

31. Reichel CA, Khandoga A, Anders HJ, Schlöndorff D, Luckow B, Krombach F. Chemokine receptors Ccr1, Ccr2, and Ccr5 mediate neutrophil migration to postischemic tissue. J Leukoc Biol. 2006;79:114-22.
32. Rothenbacher D, Müller-Scholze S, Herder C, Koenig W, Kolb H. Differential expression of chemokines, risk of stable coronary heart disease, and correlation with established cardiovascular risk markers. Arterioscler Thromb Vasc Biol. 2006;26:194-9.

33. Cavusoglu E, Eng C, Chopra V, Clark LT, Pinsky DJ, Marmur JD. Low plasma RANTES levels are an independent predictor of cardiac mortality in patients referred for coronary angiography. Arterioscler Thromb Vasc Biol. 2007;27:929-35.

34. Hirano F, Kobayashi A, Makino I. Inhibition of TNF-alpha-induced RANTES expression in human hepatocyte-derived cells by fibrates, the hypolipidemic drugs. Int Immunopharmacol. 2003;3(2):225-32.

35. Hirano Y, Hirano F, Fujii H, Makino I. Fibrates suppress chenodeoxycholic acid-induced RANTES expression through inhibition of NF-kappaB activation. Eur J Pharmacol. 2002;448(1):19-26.

36. Li S, Gokden N, Okusa MD, Bhatt R, Portilla D. Anti-inflammatory effect of fibrate protects from cisplatin-induced ARF. Am J Physiol Renal Physiol. 2005;289(2):F469-80.

37. Kitajima K, Miura S, Mastuo Y, Uehara Y, Saku K. Newly developed PPAR- $\alpha$ agonist (R)-K-13675 inhibits the secretion of inflammatory markers without affecting cell proliferation or tube formation. Atherosclerosis. 2009;203(1):75-81.

38. Fernandes KS, Béla SR, Andrade VL, et al. Simvastatin does not reduce chemokine production in obesity without comorbidities. Inflammation. 2015;38(3):1297-301.

39. Shimizu K, Aikawa M, Takayama K, Libby P, Mitchell RN. Direct anti-inflammatory mechanisms contribute to attenuation of experimental allograft arteriosclerosis by statins. Circulation. 2003;108:2113-20.

40. Mason RP, Corbalan JJ, Jacob RF, Dawoud H, Malinski T. Atorvastatin enhanced nitric oxide release and reduced blood pressure, nitroxidative stress and rantes levels in hypertensive rats with diabetes. J Physiol Pharmacol. 2015;66:65-72.

41. Piorkowski M, Fischer S, Stellbaum C, et al. Treatment with ezetimibe plus low-dose atorvastatin compared with higher-dose atorvastatin alone: is sufficient cholesterol-lowering enough to inhibit platelets? J Am Coll Cardiol. 2007;49:1035-42. 